\title{
Overcomplete Image Representations for Texture Analysis
}

\author{
Rodrigo Nava \\ Posgrado en Ciencia e Ingeniería de la Computación, Universidad Nacional Autónoma de México, Mexico City, Mexico \\ Advisor/s: Dr. Boris Escalante-Ramírez and Dr. Gabriel Cristóbal \\ Date and location of PhD thesis defense: 23th October 2013, Universidad Nacional Autónoma de México
}

Received 14th January 2014; accepted 26th May 2014

\section{Abstract}

Computer vision has played an important role in many scientific and technological areas mainly because modern society highlights vision over other senses. At the same time, application requirements and complexity have also increased, so that in many cases, the optimal solution depends on the intrinsic characteristics of the problem. Therefore, it is difficult to propose a universal image model. In parallel, advances in understanding the human visual system have allowed to suggest sophisticated models that incorporate simple phenomena. This dissertation aims to investigate characteristics of vision such as over-representation and orientation of receptive fields to develop a bio-inspired image model for texture analysis. Fourier analysis is the most common way to characterize images in the space-frequency domain, however, this type of expansion is limited. As an alternative, a more general and powerful methodology based on the so-called overcomplete methods was used. Starting from the studies of Gabor [1], Daugman [2], and Hubel and Wiesel [3], the author proposes an overcomplete image model that takes advantage of redundant information such as greater flexibility in approximations (for instance, an image can be decomposed onto multiple bases) and the increased stability of the representation, namely less sensitive to noise. Another advantage is that it is possible to explain high-dimensional data in terms of a concise set of primitive features. The equivalence between the experimental findings on orientation selectivity of visual cortical neurons and the structure of Gabor functions allow to claim that this proposal is biologically inspired.

It is well known that a Gabor expansion generates high-dimensional representations, therefore, a step where data dimension is reduced using Fisher theory and kernel methods was included. This step leads to a better characterization of visual scenes. To validate the proposal, several experiments have been successfully performed on texture segmentation [4] and classification [6]. Furthermore, a comparison with several models from the state-of-art was conducted. The last part of this dissertation states that the combination of global and local descriptors provides robust features that lead to an improvement in the description of textures. Thus, a study of local descriptors was included, specifically based on local binary patterns (LBPs) [5]. It is noticeable the use of texture patterns in medical imaging to distinguish between pathological and healthy tissue, therefore, this thesis addressed the problem of characterization of lung emphysema and proposed a novel approach that exploits the advantages Gabor filters and simultaneously encodes local information provided by LBPs [7, 8]. The results of the experiments were consistent with the theory and demonstrated the effectiveness of the proposal.

Correspondence to: <rodie@unam.mx>

Recommended for acceptance by $<$ Alicia Fornés and Volkmar Frinken $>$

ELCVIA ISSN:1577-5097

Published by Computer Vision Center / Universitat Autònoma de Barcelona, Barcelona, Spain 


\section{References}

[1] D. Gabor, "Theory of communication", J. Inst. Elec. Eng. (London) 93III:429-457, 1946.

[2] J.G. Daugman,, "Uncertainty relation for resolution in space, spatial frequency, and orientation optimized by two-dimensional visual cortical filters", J. Opt. Soc. Am. A 2:1160-1169, 1985.

[3] D.H. Hubel, T.N. Wiesel, Brain and visual perception: The Story of a 25-year Collaboration, Oxford University Press, 2005.

[4] R. Nava, B. Escalante-Ramírez, G. Cristóbal, "A comparison study of Gabor and log-Gabor wavelets for texture segmentation", 7th International Symposium on Image and Signal Processing and Analysis (ISPA), Dubrovnik, 189-194, 2011.

[5] R. Nava, G. Cristóbal, B. Escalante-Ramírez, "A comprehensive study of texture analysis based on local binary patterns", SPIE Europe Optics, Photonics, and Digital Technologies for Multimedia Applications II, Brussels, 8436:84360E, 2012.

[6] R. Nava, B. Escalante-Ramírez, G. Cristóbal, "Texture image retrieval based on log-Gabor features", Progress in Pattern Recognition, Image Analysis, Computer Vision, and Applications (CIARP), Buenos Aires, 7441:414-421, 2012.

[7] R. Nava, V. Marcos, B. Escalante-Ramírez, G. Cristóbal, L.U. Perrinet, R.S.J. Estépar, "Advances in texture analysis for emphysema classification", Progress in Pattern Recognition, Image Analysis, Computer Vision, and Applications (CIARP), Havana, 8259:214-221, 2013.

[8] R. Nava, B. Escalante-Ramírez, G. Cristóbal, R.S.J. Estépar, "Extended Gabor approach applied to classification of emphysematous patterns in computed tomography", Medical \& Biological Engineering \& Computing, 52(4):393-403, 2014.

[9] R. Nava, "Overcomplete image representations for texture analysis", Universidad Nacional Autónoma de México, 2013 [ftp://pisa.fi-p.unam.mx/www/thesis/nava_PhD.pdf] 